\title{
An Improved Method of Raga Content Assessment in Songs with Therapeutic Benefits
}

\author{
Swarima Tewari, Soubhik Chakraborty ${ }^{*}$ \\ Department of Mathematics, Birla Institute of Technology, Mesra, Ranchi-835215, India.
}

How to cite this paper: Swarima Tewari, Soubhik Chakraborty. (2020) An Improved Method of Raga Content Assessment in Songs with Therapeutic Benefits. Journal of Applied Mathematics and Computation, 4(4), 113-117.

DOI:10.26855/jamc.2020.12.001

Received: September 11, 2020

Accepted: October 5, 2020

Published: October 14, 2020

${ }^{*}$ Corresponding author: Soubhik Chakraborty, Department of Mathematics, Birla Institute of Technology, Mesra, Ranchi-835215, India.

Email: soubhikc@yahoo.co.in

\begin{abstract}
A raga, in Indian classical music, be it Hindustani or Carnatic, is a melodic structure with fixed notes and a set of rules that characterize a particular mood conveyed by performance. Ragas have well acknowledged rich emotional content (the so called raga rasa) but a common man generally lacks the aesthetic sense to understand these ragas and hence cannot derive their therapeutic benefits completely. Accordingly when it comes to music medicine and music therapy, a nice idea would be to first render songs based on a particular raga one by one with gradually increasing raga content in them and then render this specific raga itself whereby determining the raga content in a song becomes an interesting and intriguing research problem. In an earlier work, we proposed a technique relying on correlation coefficient (based on pitch that characterize a musical note) of melodic shapes, as a measure of melodic similarity, to address the issue. Considering the limitations of correlation coefficient, in the present work, we propose an improved method by incorporating more features into our analysis. These additional features include note duration, inter onset interval and pitch movements between the notes. The experimental results are encouraging.
\end{abstract}

\section{Keywords}

Music Medicine, Music Therapy, Raga, Note Duration, Inter Onset Interval, Pitch

\section{Introduction}

Music may be defined as an emotive, acoustical and cooperative communication, universal in nature [1]. The tacit usage of the word universal separates music from speech. For speech, which primarily conveys a message unlike music which primarily conveys emotion, it is important to know the language. While music also has its language and alphabets (the musical notes or beats), one can still enjoy music without knowing them thereby rendering it a universal appeal.

Indian classical music (ICM) has two broad components: North Indian or Hindustani and South Indian or Carnatic forms. In either form, the nucleus is the raga. A raga, in Indian classical music, be it Hindustani or Carnatic, is a melodic structure with fixed notes and a set of rules that characterize a particular mood conveyed by performance [2]. For a sound literature on Hindustani ragas, we recommend the book [3]. Ragas have well acknowledged rich emotional content (the so called raga rasa) but a common man generally lacks the aesthetic sense to understand these ragas and hence cannot derive their therapeutic benefits completely. Accordingly when it comes to music medicine and music therapy, a nice idea would be to first render songs based on a particular raga one by one with gradually increasing raga content in them and then render this specific raga itself whereby determining the raga content in a song becomes an interesting and intriguing research problem. In an earlier work (see section 2), we proposed a technique relying on correlation coefficient (based on pitch that characterize a musical note) of melodic shapes, as a measure of melodic similarity, to address the issue. Considering the limitations of correlation coefficient, in the present work we propose an improved method by incorporating more features into 
our analysis. These additional features include note duration, inter onset interval and pitch movements between the notes.

\section{Measuring raga content using correlation coefficient}

Two melodies of equal length are statistically similar if the correlation coefficient of their shapes is statistically found significant, using a standard t-test. To understand this definition, we need to understand a melody, its length and shape, correlation coefficient and its importance and limitations in the context and the standard t-test to test the significance of an observed sample correlation coefficient. Melody is that part of music which is pleasant and rhythm is that part of music which is periodic. But this definition of melody does not allow statistical analysis directly. So we alternatively define melody as a sequence of notes (characterized by pitch) which is complete to be a musical sentence or at least a musical phrase. For example each line of a song being complete is a melody. A Segment is an incomplete sequential subset of the melody. By length of a melody or its segment, we simply mean the total number of notes in it [4]. Indian music is monophonic implying a single melody line in which the significance of a melody (or its segment) is evaluated by multiplying its length with the number of times (frequency) it occurs in the musical piece [5] while for polyphonic music (multiple melody lines) this significance is evaluated using the method given in [6]. By the shape of a melody, we mean the difference of successive pitches of the notes in it. The correlation coefficient $r$ of the shapes for two melodies of equal length is a measure of similarity between the corresponding melodies in the sense that if the value of $r=1,-1$ or 0 , the two melodies will be in translation, in inversion or different respectively.

For computing the raga \% in the song, segments and melodies of equal length will be compared with those from a standard database of the raga concerned [7].

Significance of an observed correlation coefficient can be tested using t-test. Thus, we are testing the null hypothesis $\mathrm{H}_{0}$ that the population correlation coefficient is zero against the alternative hypothesis $\mathrm{H}_{1}$ that it is non zero. Let $\mathrm{r}=$ sample correlation coefficient and $\mathrm{n}=$ number of pairs of observations (here successive differences of pitch). Then our test statistic is $t=r \frac{\sqrt{(n-2)}}{\sqrt{\left(1-r^{2}\right)}}$ which follows Student's $t$ distribution with $(\mathrm{n}-2)$ degrees of freedom. If calculated $|\mathrm{t}| \geq$ table value of $\mathrm{t}$ at $5 \%$ level of significance (say) and (n-2) degrees of freedom, then the value of $r$ is significant at $5 \%$ level, in which case $\mathrm{H}_{0}$ is rejected at $5 \%$ level, otherwise insignificant in which case we may accept $\mathrm{H}_{0}$. Here it is assumed that the $\mathrm{n}$ pairs are coming from a bivariate normal distribution. The formula for $\mathrm{r}$ is $\frac{\operatorname{covariance}(x, y)}{\{s d(x) * s d(y)\}}$ where sd = standard deviation. Covariance (x, y) can be computed easily as $\left\{\frac{\sum(x * y)}{n}\right\}-\{\bar{x} * \bar{y}\} ; s d(x)=+\sqrt{\left[\left\{\sum\left(\frac{x * x}{n}\right)\right\}-\{\bar{x} * \bar{x}\}\right]}$ and similarly sd(y) replacing $\mathrm{x}$ by $\mathrm{y}$ in the formula for $\operatorname{sd}(\mathrm{x}) \cdot \bar{x}=\frac{\sum(x)}{n}$ and $\bar{y}=\frac{\sum(y)}{n}$, the summation extending over all the $\mathrm{n}$ values of $\mathrm{x}$ or $\mathrm{y}$. Since the $\mathrm{t}$ statistic has (n-2) degrees of freedom, we must have $n>2$. This means melodies of lengths at least 4 (whose shapes will have at least $\mathrm{n}=3$ successive pitch values) can be compared for similarity [5].

In our study, we have compared melodies and segments of lengths 4, 5 and 6 only and a separate \% of similarity is obtained. Then a weighted mean of this raga \% is taken, the weights being the corresponding lengths. The algorithm being used picks up melodies or segments of a specified length from the song, one at a time, and compares with every melody or segment of similar length from the raga note sequence in the data base [7].

A counter initialized at zero is used. If a single comparison yields similarity we would increase the counter by one, stop any further comparison of this melody or segment and pick up the next one from song sequence. However, if the next melody or segment, or any other from the song note sequence picked likewise, turns out to be identical to a previous one in the song that was found similar with that in the raga, then we agree to increase the counter by one as we have already considered the number of times a melody or segment is repeated as an important factor in assessing its significance. In short, all repeated song melodies or segments, if found similar with the raga, will contribute towards raga \%. While ragas are very rich in their emotional content, a common man may not have the receiving (understanding) capacity and hence cannot derive its therapeutic value fully. So a good idea is to render raga based songs first in gradually increasing order of raga content and then render the raga itself. The strategy has been successfully implemented in the context of patients with cerebrovascular accident and diffuse head injury [8]. Therefore, evaluating the raga content in the song becomes a research problem [9].

\section{Moving towards an improved measure of raga content}

Since correlation coefficient considers only pitch values of notes and neglects several other features, to be discussed and incorporated now, it is limited in measuring melodic similarity. It is quite possible that two statistically similar melodies will be dissimilar musically (as found by listening to them) just as two musically similar sounding melodies could be statistically dissimilar. 
The discrepancy arises by other musical features neglected in computing the correlation coefficient. These other features comprise of note duration, pitch movements between the notes, inter onset interval. Let us briefly describe them one by one. Note duration is important as herein lies the concept of psychological stability of a note. If we stay on a note, it gives a sense of rest or psychological stability. Why we are calling this pitch stability psychological is to distinguish it from another concept of pitch stability which is called statistical pitch stability based on the stability of relative occurrences of pitch over the instances of their realization in the musical pitch [5]. Pitch movements between the notes is important because how we are moving from one note to another-whether ascending or descending-and in either case whether the pitch movement is linear, convex or concave which will determine the contrasting emotions [10]. Finally, a set of successive notes are in rhythm if they have the same inter onset interval (IOI), i.e., the time interval between the occurrences of two successive notes is equal [5]. Observe that note duration and IOI are not the same. Note duration is the difference between departure time and arrival time of the same note. IOI for two successive notes is the difference between the arrival time of the next note and the arrival time of the present note.

Additionally, it is always wise to have the songs graded by a musician also, in which the musician will be asked to provide only the order of the songs rather than give any subjective value of raga content measures, and then test the significance of the rank correlation coefficient between the musician's ranking and the scientific ranking since, from a therapeutic angle, it is the order ultimately rather than the raga content measure that is of interest. The doctor treating the patient only needs to know this order. We must bear in mind the limitation of the correlation coefficient which only measures the degree of linear relationship and that too for melodies of equal length. Assuming that no two songs in the list are ranked equal, let $\mathrm{X}$ and $\mathrm{Y}$ denote the ranks given by a scientist and a musician respectively to a particular song ( $\mathrm{X}$ and $\mathrm{Y}$ can be equal). $\mathrm{X}$ and $\mathrm{Y}$ both can take values (ranks) 1, 2...n for a list of $\mathrm{n}$ raga based songs (all based on the same raga). Spearman's rank correlation coefficient is calculated by the formula $\rho=1-6 \sum d^{2} / n\left(n^{2}-1\right)$ where $d=X-Y$. The significance can be tested in the same manner as in the case of $r$ replacing $r$ by $\rho$ and performing a t-test as described previously [9].

Remark: Other statistical features include statistical modeling and pitch velocity implying the rate of change of pitch which has been used recently to distinguish restful and restless ragas [11]. Statistical modeling has also been used by other researchers (see e.g. [12], [13], [14].)

\title{
4. Experimental Results
}

The following six songs were selected in our analysis.

\author{
Song 1: Chingari Koi Bhadke \\ Singer: Kishore Kumar \\ Movie: Amar Prem \\ Music composer: R. D. Burman \\ Lyricist: Anand Bakshi
}

\author{
Song 2: Mere Mann Ki Ganga \\ Singer: Mukesh \\ Movie: Sangam \\ Music composer: Shankar Jaikishan \\ Lyricist: Shailendra
}

Song 3: Jyot se JyotJagataChalo

Singer: Mukesh/Lata Mangeshkar (independently)

Movie: Sant Gyaneshwar

Music Composer: Laxmikant-Pyarelal

Lyricist: Bharat Vyas

Song 4: Khilona Jan Kar

Singer: Mohammad Rafi

Movie: Khilona

Music composer: Laxmikant-Pyarelal

Lyricist: Anand Bakshi

Song 5: LagaChunari Mein Daag

Singer: Manna Dey

Movie: Dil Hi To Hai

Music Composer: Roshan 
Lyricist: SahirLudhianvi

\section{Song 6: PhoolGendwa Na Maro}

Singer: Manna Dey

Movie: Dooj Ka Chand

Music Composer: Roshan

Lyricist: Sahir Ludhianvi

We would calculate the rank correlation coefficient for the following data and also test its significance (lowest rank indicates highest raga content).

Song: Chingari Koi Mere Mann Ki Jyot se Jyot Khilonajankar LagaChunari Phool Gendwa

$\begin{array}{lllllll}\begin{array}{l}\text { Rank: } \\ \text { (scientific) }\end{array} & 6 & 4 & 3 & 5 & 1 & 2 \\ \begin{array}{l}\text { Rank: } \\ \text { (musician A) }\end{array} & 5 & 3 & 4 & 6 & 1 & 2 \\ \begin{array}{l}\text { Rank: } \\ \text { (musician B) }\end{array} & 6 & 5 & 3 & 4 & 1 & 2\end{array}$

Let us first calculate the rank correlation coefficient between the scientific ranking and the ranking of musician A. Our calculations are shown in Table 1.

Table 1. Calculation of rank correlation coefficient for the scientific ranking and the ranking of musician $A$

\begin{tabular}{ccccc}
\hline Song & Scientific Ranking $=\mathrm{x}$ & Ranking of Musician A = & $\mathrm{d}=\mathrm{x}-\mathrm{y}$ & $\mathrm{d}^{2}$ \\
\hline Chingari Koi Bhadke & 6 & 5 & 1 & 1 \\
Mere Mann Ki Ganga & 4 & 3 & 1 & 1 \\
Jyot Se JyotJagateChalo & 3 & 4 & -1 & 1 \\
Khilona Jan Kar & 5 & 6 & -1 & 1 \\
LaagaChunari Mein Daaag & 1 & 1 & 0 & 0 \\
PhoolGendwa Na Maro & 2 & 2 & 0 & 0 \\
\hline
\end{tabular}

As there are six songs we have six pairs of ranks $(\mathrm{x}, \mathrm{y})$ and hence $\mathrm{n}=6$

Rank correlation coefficient $=1-6 \Sigma \mathrm{d}^{2} /\left(\mathrm{n}^{3}-\mathrm{n}\right)=1-(6 \mathrm{x} 4) /(216-6)=0.885714$

Calculated $\mathrm{t}=0.885714 \times \sqrt{ }(6-2) / \sqrt{ }(1-0.784489)=3.815833$

Table $t$ at $5 \%$ level of significance and $6-2=4$ degrees of freedom $=2.78$

Result: The calculated $|\mathrm{t}|$ is greater than the table $t$ and hence significant at $5 \%$ level of significance. This meansthe calculated (sample) rank correlation coefficient is significant implying that the scientific ranking is sufficiently close to the ranking of musician A.

Next we would calculate the rank correlation coefficient between the scientific ranking and the ranking of musician B. Our calculations are shown in Table 2.

Table 2. Calculation of rank correlation coefficient for the scientific ranking and the ranking of musician $B$

\begin{tabular}{ccccc}
\hline Song & Scientific Ranking $=\mathrm{x}$ & Ranking of Musician B = y & $\mathrm{d}=\mathrm{x}-\mathrm{y}$ & 0 \\
\hline Chingari Koi Bhadke & 6 & 6 & -1 & 0 \\
Mere Mann Ki Ganga & 4 & 5 & 0 & 1 \\
Jyot Se JyotJagateChalo & 3 & 3 & 1 & 0 \\
Khilona Jan Kar & 5 & 4 & 0 & 0 \\
LaagaChunari Mein Daaag & 1 & 1 & 0 & 0 \\
PhoolGendwa Na Maro & 2 & 2 & & $\Sigma \mathrm{d}^{2}=2$
\end{tabular}

Rank correlation coefficient $=1-6 \Sigma \mathrm{d}^{2} /\left(\mathrm{n}^{3}-\mathrm{n}\right)=1-(6 \mathrm{x} 2) /(216-6)=0.942857$

Calculated $\mathrm{t}=0.942857 \times \sqrt{ }(6-2) / \sqrt{ }(1-0.888979)=5.659440$ 
Table $t$ at $5 \%$ level of significance and 4 degrees of freedom $=2.78$

Result: The calculated $|\mathrm{t}|$ is greater than the table $\mathrm{t}$ and hence significant at $5 \%$ level of significance. This means the calculated (sample) rank correlation coefficient is significant implying that the scientific ranking is sufficiently close to the ranking of musician B.

\section{Concluding remarks}

In both the experimental studies, our null hypothesis that the rank correlation coefficient is zero in the population is rejected at $5 \%$ level of significance as the sample rank correlation coefficient turned out to be significant. Thus, the scientific (logical) ranking and the musician's (intuitive) ranking are not significantly different. Hence our improved proposed technique of raga content measurement seems to be working. However, our research team is working on further improvements by incorporating more musical features in our analysis. It should be understood that the ultimate aim of our study is to provide therapeutic benefits of music to the patients in some optimum sense.

\section{Acknowledgement}

We thank two musicians, who wish to remain anonymous, for supporting this research.

\section{References}

[1] Datta, A. K., Solanki, S. S., Sengupta, R., Chakraborty, S., Mahto, K., and Patranabis, A. (2017). Signal Analysis of Hindustani Classical Music, Springer.

[2] Chakraborty, S., Ranganayakulu, R., Chauhan, S., Solanki, S. S., and Mahto, K. (2009). A Statistical Analysis of Raga AhirBhairav. Journal of Music and Meaning, Vol. 8, sec. 4, http://www.musicandmeaning.net/issues/showArticle.php?artID=8.4.

[3] Jairazbhoy, N. A. (1995). The Ragas of North Indian Music: Their Structure \& Evolution. Bombay: Popular Prakashan.

[4] Chakraborty, S., Krishnapryia, K., Loveleen, Chauhan, S., Solanki, S. S., and Mahto, K. (2010). Melody Revisited: Tips from Indian Music Theory. International Journal of Computational Cognition, Vol. 8(3), 26-32.

[5] Chakraborty, S., Mazzola, G., Tewari, S., and Patra, M. (2014). Computational Musicology in Hindustani Music. Springer.

[6] Adiloglu, K., Noll, T., and Obermayer, K. A. (2006). Paradigmatic Approach to Extract the Melodic Structure of a Musical Piece. Journal of New Music Research, Vol. 35(3), 221-236.

[7] Dutta, D. (2006). Sangeet Tattwa, Pratham Khanda (in Bengali), BratiPrakashani, $5^{\text {th }}$ ed.

[8] Singh, S. B., Chakraborty, S., Jha, K. M., Chandra, S., Prakash, S., and Tewari, S. (2016). Music and Medicine: Healing Brain Injury Through Ragas. CBH publications.

[9] S. Tewari and S. Chakraborty. (2020). Modeling a Raga-Based Song and Evaluating its Raga Content: Why it Matters in a Clinical Setting, S. K. Sahana and V. Bhattacharjee (eds.), Advances in Computational Intelligence, Advances in Intelligent Systems and Computing 988, https: //doi.org/10.1007/978-981-13-8222-2_21, pp. 255-264, Springer Nature Singapore Pte Ltd.

[10] Tewari, S. and Chakraborty, S. (2011). A Statistical Analysis of Raga Bhairavi. Acoustic Waves, S. K. Srivastava, Kailash, K. Chaturvedi (Ed.), Shree Publishers and Distributors, New Delhi, pp. 329-336.

[11] Priyadarshini, P. and Chakraborty, S. (2017). Using Statistical Modeling, Rate of Change of Pitch and Inter Onset Interval to Distinguish Between Restful and Restless Ragas. Communications in Mathematics and Statistics, Volume 5(2), pp. 199-212.

[12] Beran, J. and Mazzola, G. (1999). Analyzing musical structure and performance- a statistical approach. Statistical Science, Vol. 14 (1), 47-79.

[13] Pearce, M. T., Wiggins, G. A. (2004). Improved Methods for Statistical Modelling of Monophonic Music. Journal of New Music Research. Vol. 33, Article 4, pp. 367-385.

[14] Wiggins, G. A., Pearce, M. T., and Müllensiefen, D. (2011). Computational Modeling of Music Cognition and Musical Creativity. The Oxford Handbook of Computer Music edited by R. T. Dean, Oxford University Press. 